\title{
Nasal Fractures: The Role of Primary Reduction and Secondary Revision
}

\author{
Weitao Wang, MD ${ }^{1}$ Thomas Lee, MD, FACS ${ }^{2}$ Scott Kohlert ${ }^{3}$ Sameep Kadakia, MD ${ }^{4}$ \\ Yadranko Ducic, MD, FRCS(C), FACS ${ }^{3}$ \\ ${ }^{1}$ Department of Otolaryngology, University of Rochester Medical \\ Address for correspondence Weitao Wang, MD, Department of \\ Center, Rochester, New York \\ Otolaryngology, University of Rochester Medical Center, 601 \\ 2 Department of Otolaryngology, Howard Hughes Medical Institute, \\ Virginia Commonwealth University School of Medicine, Richmond, \\ Elmwood Ave., Box 629, Rochester, NY 14642-8629 \\ Virginia \\ ${ }^{3}$ Otolaryngology and Facial Plastic Surgery Associates, Fort Worth, \\ (e-mail: Weitaow@gmail.com). \\ Texas \\ ${ }^{4}$ Department of Surgery, Wright State University, Dayton, Ohio \\ Facial Plast Surg 2019;35:590-601.
}

\begin{abstract}
Keywords

- nasal fracture

- closed reduction

- nasal trauma

- septorhinoplasty

- septoplasty

The nasal bones are among the most commonly fractured bones in the facial skeleton. Proper management of nasal trauma acutely is important in minimizing secondary deformities and impaired function with nasal airway obstruction. Septal hematoma, if present, should be drained right away. Acutely closed nasal reduction and limited septoplasty can be performed. Unrecognized septal fracture may play a role in the failure of closed nasal reduction of fractured nasal bones. Complex nasoorbitoethmoid fractures are approached openly and treated with rigid fixation. Primary use of open rhinoplasty in an acute setting is debated, and there are no clearly accepted indications for timing, patient selection, and surgical technique. However, open septorhinoplasty is more commonly used in a delayed fashion to provide definitive correction of any residual cosmetic or functional problems. Recent algorithms provide a systematic approach to nasal trauma and may improve secondary deformity rates following closed reduction.
\end{abstract}

The nasal bones are one of the most frequently fractured bones in the facial skeleton. Studies have shown the incidence to be as high as $40 \%$ of all facial fractures. ${ }^{1}$ Many algorithms have been proposed to approach nasal bone fractures to achieve optimal functional and cosmetic outcomes. Nonetheless, there remain high rates of posttraumatic deformity requiring open septorhinoplasty. Watson et al and Waldron et al described secondary nasal deformities in those who underwent primary closed nasal reduction to be between 14 and 50\%. Murray and Maran in the largest prospective study of 756 patients reported a $41 \%$ postreduction deformity rate of $41 \%{ }^{2-4}$ A recent population study suggested those with preexisting nasal obstruction or defect were associated with higher rates of revision septorhinoplasty. ${ }^{5}$ The literature is reviewed here regarding the optimal approach to manage isolated nasal bone fractures.

\section{Nasal Anatomy and Force Considerations}

\section{Nasal Anatomy}

The nasal framework can be broken up into thirds (- Fig. 1A). The upper third consists of nasal bones, which are thin bones of approximately $2.5 \mathrm{~cm}$ in length but variable in length in the adult, and extend caudally toward the rhinion, the junction between the upper lateral cartilage and nasal bone (-Fig. 1B). This is a critical area and is sometimes referred to as the "keystone area," where the septum is also secured to this area for stability. Superiorly, the radix (also known as nasion) marks the nasofrontal bony junction. The middle third comprises of the paired upper lateral cartilages, which attach to the septum at midline, providing structural support to the middle vault and is part of the internal nasal
Issue Theme Contemporary Management of Facial Trauma and Complications; Guest Editor: Yadranko Ducic, MD, FRCS(C), FACS
Copyright (c) 2019 by Thieme Medical Publishers, Inc., 333 Seventh Avenue, New York, NY 10001, USA. Tel: +1(212) 584-4662.
DOI https://doi.org/ 10.1055/s-0039-1700801. ISSN 0736-6825. 
valve, which is critical for nasal airflow. The lower third includes the paired lower lateral cartilages spanning from the columella toward the alar crease, which provides aesthetic contour to the nasal tip and is part of the external nasal valve (-Fig. 1B). The nasal septum plays a vital role in maintaining the structure of the nose, especially along the middle one-third (-Fig. 2). The septum is composed of the quadrangular cartilage anteriorly. A combination of the vomer, perpendicular plate of the ethmoid, maxillary crest, and palatine bones form the posterior portion of the septum. ${ }^{6}$

\section{Fracture Patterns}

In analyzing trends in causes of fracture, Kim et al found no significant difference in incidence but increase in severity of nasal bone fractures due to traffic accidents. Common causes include assault, fall, and traffic accidents. ${ }^{7}$ Previous studies showed that in $46.9 \%$ of nasal bone fractures have concomitant septal fracture. ${ }^{8}$ The senior author (Y. D.) previously described three types of septal fracture patterns including isolated cartilaginous, single fracture through the osseocartilaginous junction, and multiple fractures at any location occurring at progressively higher amounts of force. ${ }^{9}$

\section{Diagnosis}

Nasal bone fractures can occur in isolation or in conjunction with frontal, nasoorbitoethmoid (NOE), or midfacial fractures. Initial management should include stabilization of the airway, breathing, and circulation, as with any trauma patient. Following this, history is noted and physical examination is performed. A thorough examination of the nasal bones begins with visual evaluation of the upper one-third for collapse or deviation of nasal bones. Along the middle one-third, upper lateral cartilage detachment from the septum can result in an inverted V-deformity, internal nasal valve collapse, and contour irregularity along the midvault. Along the lower one-third, caudal septal deflections, columella retraction or deviation, nasal tip deviation, and dynamic alar collapse with inspiration from weak internal or external nasal valve are assessed.

Anterior rhinoscopy should be performed to evaluate the status of the septum to rule out septal hematoma and to

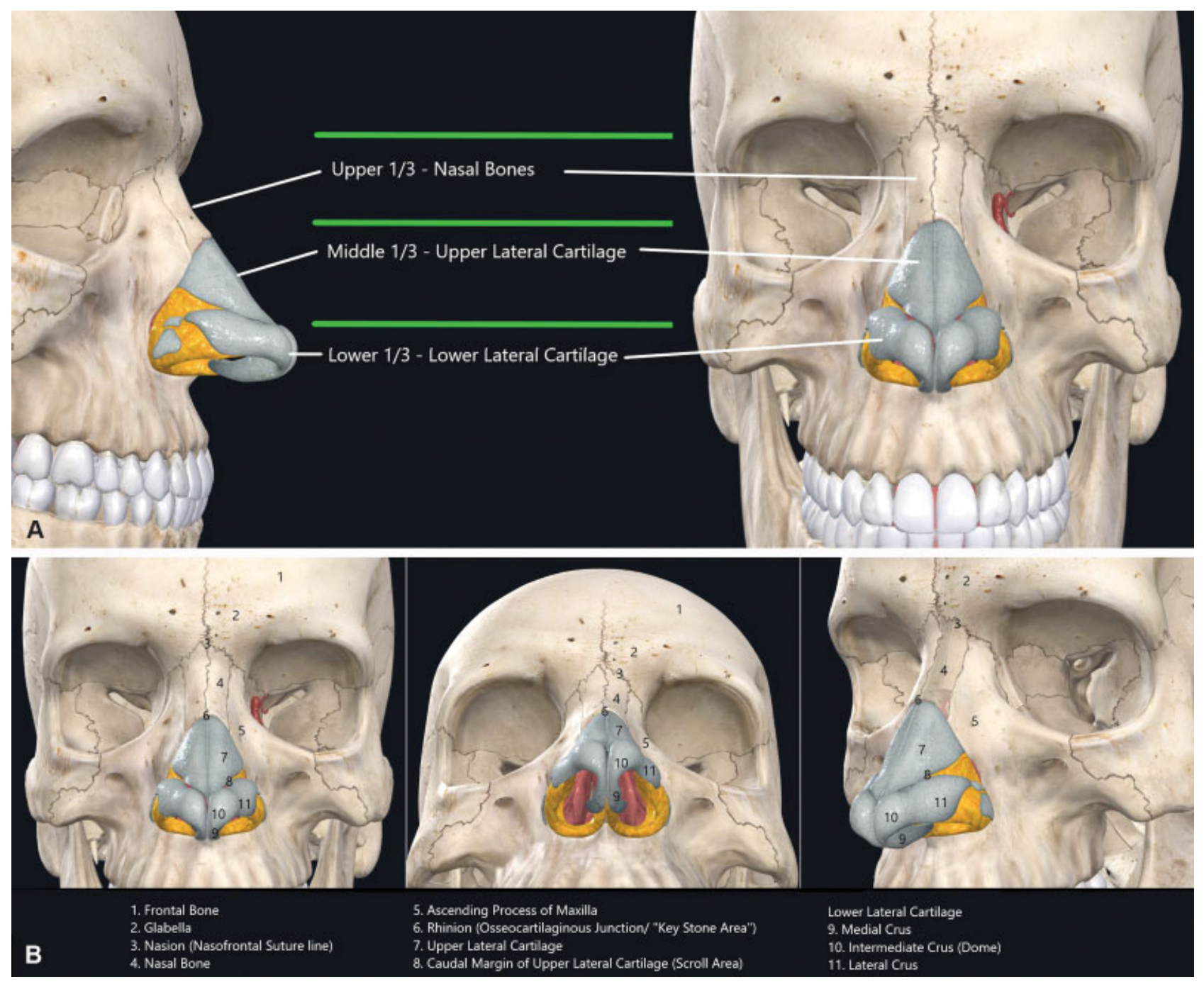

Fig. 1 (A) Nose is analyzed in thirds. Nasal bones are located in upper one-third. Upper lateral cartilage is located in the middle one-third. Lower lateral cartilage is located in the lower one-third. (B) External anatomy of the nose is labeled. 




Fig. 2 Septum anatomy. Left oblique and sagittal views demonstrate the relationship between the quadrangular cartilage, maxillary crest, perpendicular plate of the ethmoid, and vomer in relation to the nasal bones. The cartilaginous septum is located along the lower two-thirds and is prone to saddle-nose deformity with improper septal support. The bony septum is located below the nasal bones. The keystone are links the septum to the nasal bone and must be preserved for adequate stability. To maintain septal support, 1 to $1.5 \mathrm{~cm}$ of septal cartilage must be preserved as an L-strut (green lines) during septoplasty.

evaluate the position of the internal nasal valve. Rohrich and Adams proposed a thorough endoscopic evaluation of the nose as part of the initial examination, although endoscopic visualization of posterior airway maybe challenging in acute trauma due to a significant amount of bleeding and mucosal edema likely present. ${ }^{10}$ Imaging is generally not useful for isolated nasal bone fractures. When possible, a photograph of the patient's preinjury state should be assessed to evaluate for any preexisting deformity prior to injury. There is a role for imaging in cases with high suspicion for concomitant facial fractures based on the remainder of the examination of the facial skeleton. Plain films are not useful in these cases, and computed tomography (CT) maxillofacial scans without contrast are preferred.

Attempts at classifying nasal bone fractures date back to Gilles in 1929 where a system was based on impact direction. ${ }^{11}$ Stranc and Robertson in 1979 based their system on injury planes. ${ }^{12}$ Murray and Maran in 1990 classified seven fracture types based on anatomy in cadavers. ${ }^{13}$ Rohrich and Adams described an anatomy-based classification of six types. ${ }^{10}$ No one system of classification is routinely used, and reported systems in the literature lack uniformity. The ultimate goal of an ideal classification is its effect on prognosis following treatment, allowing for an optimal algorithm yielding the highest excellent results.

\section{Management}

The primary goals in the treatment of isolated nasal bone fractures are premorbid function restoration and cosmesis. Given the variably high rates of revision septorhinoplasty in the literature, a secondary goal of treatment would be to minimize posttreatment deformity. A thorough nasal analysis including intranasal evaluation must be completed at the time of evaluation. Septal hematoma can present as boggy-appearing mucosa that is fluctuant upon palpation with a Q-tip or suction. Patients may have nasal obstruction from septal mucosal swelling out laterally and contacting the inferior turbinates. Any septal hematoma should be drained immediately to avoid septal cartilage necrosis, infection or saddle-nose deformity (-Fig. 3).

In the literature, there is no clear consensus on the best treatment algorithm in an acute trauma setting. There have been many attempts at streamlining the approach to the management of nasal bone fractures. The options for primary management in an acute setting include the following:

- Observation.

- Closed nasal reduction.

- Closed nasal reduction with limited septoplasty.

- Open septorhinoplasty with ear cartilage or rib cartilage.

- Open reduction internal fixation of nasal bones/NOE fractures \pm septorhinoplasty

In the acute setting, aside from closed nasal reduction and laceration repair, most practitioners tend to avoid performing definitive open septorhinoplasty (option 4) that requires extensive dissection and cartilage grafting. The trend is to perform conservative procedures acutely and wait for 3 to 6 months prior to considering a definitive open septorhinoplasty once all of the soft tissue trauma and cartilage contracture forces have stabilized to more accurately reflect long-term appearance and nasal airflow.

The reasoning for initial conservative, less invasive treatment in the acute setting is that there are several variables 


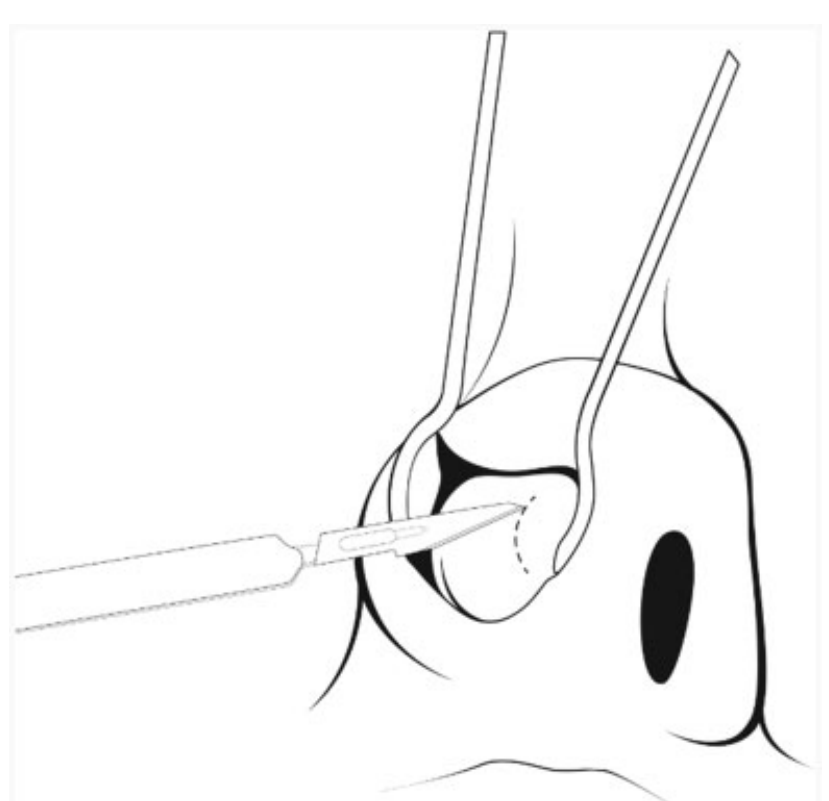

Fig. 3 Septal hematoma/abscess drainage. Anterior rhinoscopy is performed and a no. 11 blade is used to incise the mucoperichondrium and allow drainage of contents to prevent septal cartilage necrosis. Once the hematoma is drained, a small drain can be sutured to keep the incision open for approximately 3 to 5 days or longer for ongoing irrigation if infected.

that can influence the final, long-term outcomes both cosmetically and functionally. First, if there is a laceration involving the nasal skin, the traditional open rhinoplasty approach can severely compromise the skin envelope vascularity and may result in exposed nasal cartilages if the skin flap dies. Furthermore, the scar can thin out greatly over time and can give the illusion of saddle-nose deformity even if the underlying cartilage is intact, which may require subcutaneous grafting under the thin scar to provide desirable skin contour. A scar can also become hypertrophic and provide excess volume to the nasal dorsum or highlight asymmetry. All scars take time to mature and may improve in appearance naturally with time. Long-term appearance of facial scars cannot be reliably assessed until approximately 6 to 12 months after the accident. Second, if the nasal cartilages have been transected, it is best to avoid performing any significant dissection as lifting the skin or underlying nasal mucosa away from the cartilage, which is typically required in cartilage grafting, as this can devascularize the nasal cartilage. In the acute setting, it is best to reapproximate the transected nasal cartilages with sutures and close the overlying and skin and the nasal mucosa in a layered fashion. Transected cartilages will likely undergo a varying degree of scarring and weakening, and this area may require cartilage grafting to provide adequate support at the time of definitive nasal surgery. Third, similarly, if the nasal bones are severely disrupted with an open laceration, it is imperative that the nasal bones are not exposed and the overlying skin envelope must be repaired. If the nasal bones are present in combination with concurrent upper or midface fractures, it may require open reduction and internal fixation with hardware using the existing laceration for approach. Modified lynch incisions can be used to access the NOE fracture site along the upper one-third, whereas bicoronal incision can be used if there is a concurrent frontal sinus fracture that will require repair. Fourth, in the setting of a dirty, infected wound, it is best to avoid performing any cartilage grafting as any cartilage graft will likely necrose and make the infection worse. Cartilage grafts rely on surrounding skin and mucosal envelope for blood supply. As such, definitive rhinoplasty requiring cartilage grafting cannot occur in a reliable fashion when an infection is present. In such a scenario, any abscess present should be drained and the patient should be treated conservatively with antibiotics. It is generally advisable to defer definitive correction septorhinoplasty for several months until all the infection has been resolved.

In the literature, most authors recommend initial conservative approach with closed nasal reduction and limited septoplasty in the acute setting with a plan for delayed definitive correction septorhinoplasty depending on the outcome. Basheeth et al reported in their prospective study that 191 out of 400 patients required closed nasal reduction, while others were treated with initial observation alone. ${ }^{14}$ Rohrich and Adams proposed an algorithm in which patients with noncomminuted, unilateral or bilateral, and comminuted nasal bones underwent fracture reduction after overlying skin edema subsides. In cases in which septal fracture was present, reduction of septal fractures and conservative septoplasty were considered. Contrastingly, patients with nasal bones fractures with concurrent NOE, frontal sinus, or LeFort fractures underwent early open reduction internal fixation. They reported a revision rate of $9 \%$ in 110 patients, which is one of the lowest reported in the literature. ${ }^{10}$

Hoffmann described a more simplified algorithm. He recommends that closed reduction should be performed in unilaterally/bilaterally displaced or mildly comminuted nasal bone fractures with mild septal deviation. Bilateral comminuted nasal fractures with moderate septal deviation should be treated with closed nasal reduction, with closed septal reduction or limited septoplasty. Severe comminution and impacted nasal bone fractures, with severe septal disruption and saddle deformities, should be treated with closed nasal reduction, limited osteotomies, limited septoplasty, and bone grafting. Formal septorhinoplasty is reserved in all cases as needed for 3 to 6 months postoperatively. ${ }^{15}$ Ondik et al proposed a similar simplified algorithm with a revision rate of $9 \% .{ }^{16}$

Based on existing algorithms, we describe our treatment approach to also include revision options following the initial repair (-Fig. 4).

\section{Timing of Intervention}

Most argue for early reduction in the pediatric population given faster healing, generally within 7 days, and 10 days of injury for adults. ${ }^{10}$ Time to surgery has not shown to be a relevant factor in predicting long-term surgical outcomes in pediatric patients in multiple studies. Lee and Jang showed similar cosmetic outcomes in the pediatric age group who underwent treatment less than or greater than 7 days after injury. ${ }^{17}$ Yoon and Han reviewed 10 patients, with a mean 


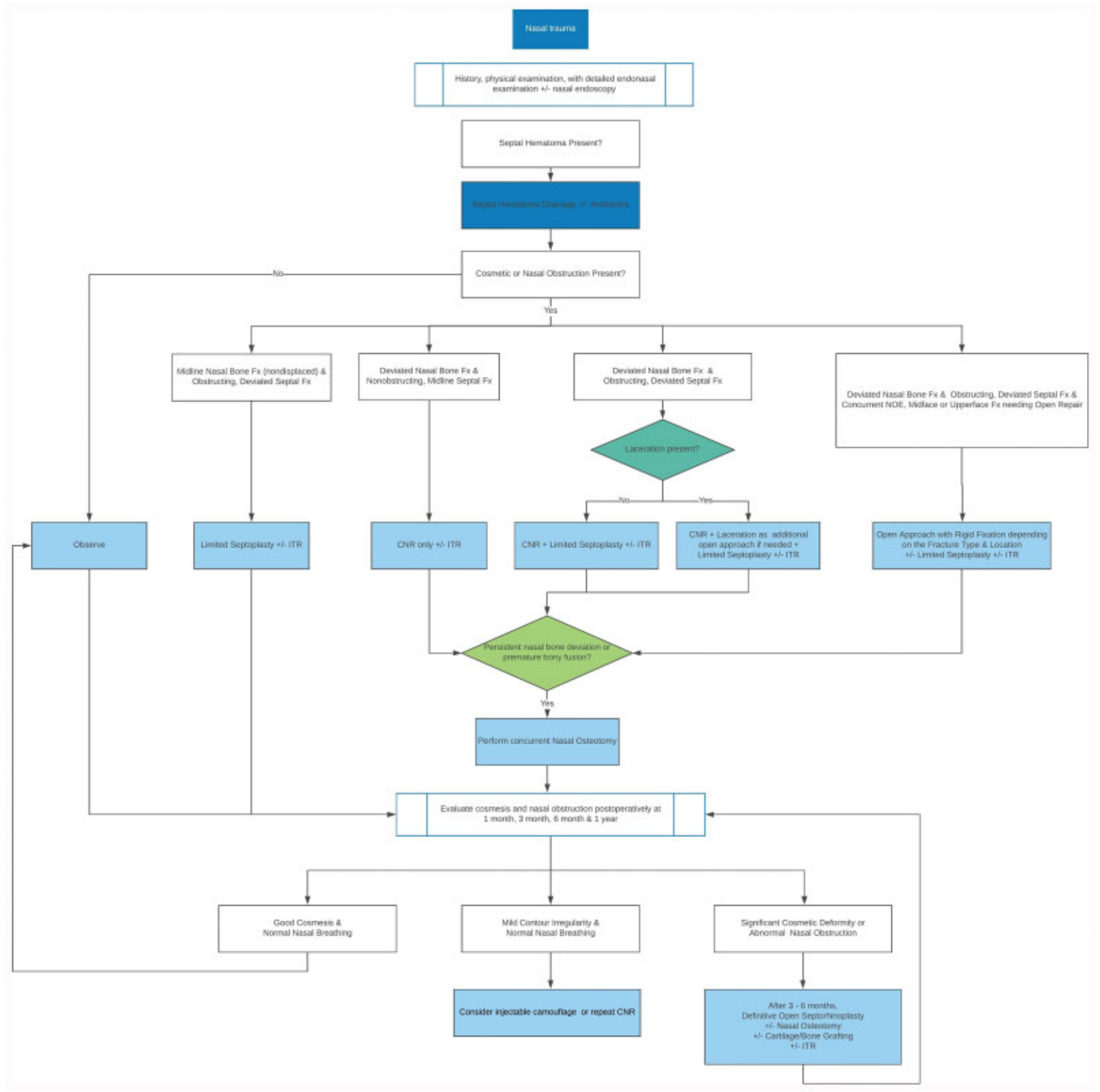

Fig. 4 Treatment algorithm of nasal bone and septal fractures after acute trauma.

trauma-to-reduction time of 22 days with good or excellent outcomes postoperatively. They suggest that a window of up to 2 to 4 weeks is adequate for closed reduction. ${ }^{18}$ Basheeth et al report having success with primary closed manipulation of the nasal bones up to 5 weeks postinjury, with a postreduction deformity rate of $22.5 \% .{ }^{14} \mathrm{Li}$ et al similarly did not find delay to fracture repair to be an associated risk factor for secondary rhinoplasty in their population study. ${ }^{5}$

In summary, closed nasal reduction can be safely performed in the acute setting. If there is a troubling skin swelling present that makes intraoperative evaluation of underlying nasal bones difficult, it is acceptable to defer closed nasal reduction for 7 to 10 days or longer to allow the skin swelling to resolve, but ideally before the bony fusion occurs. If the bony fusion has already occurred, closed nasal reduction may also require concurrent nasal osteotomy to mobilize the displaced bone segment.

\section{Closed Nasal Reduction in the Acute Setting}

Closed nasal reduction is a simple outpatient procedure that can be performed under local anesthesia or brief general anesthesia and thus is a cost-effective approach to nasal bone fractures. Performing open septorhinoplasty as the initial procedure is complicated by the acute swelling from trauma, as well as the potential lack of viable septal cartilage use given variable septal fracture patterns, making rhinoplasty results less precise. While studies have shown the closed nasal reduction can be performed under local anesthesia, 


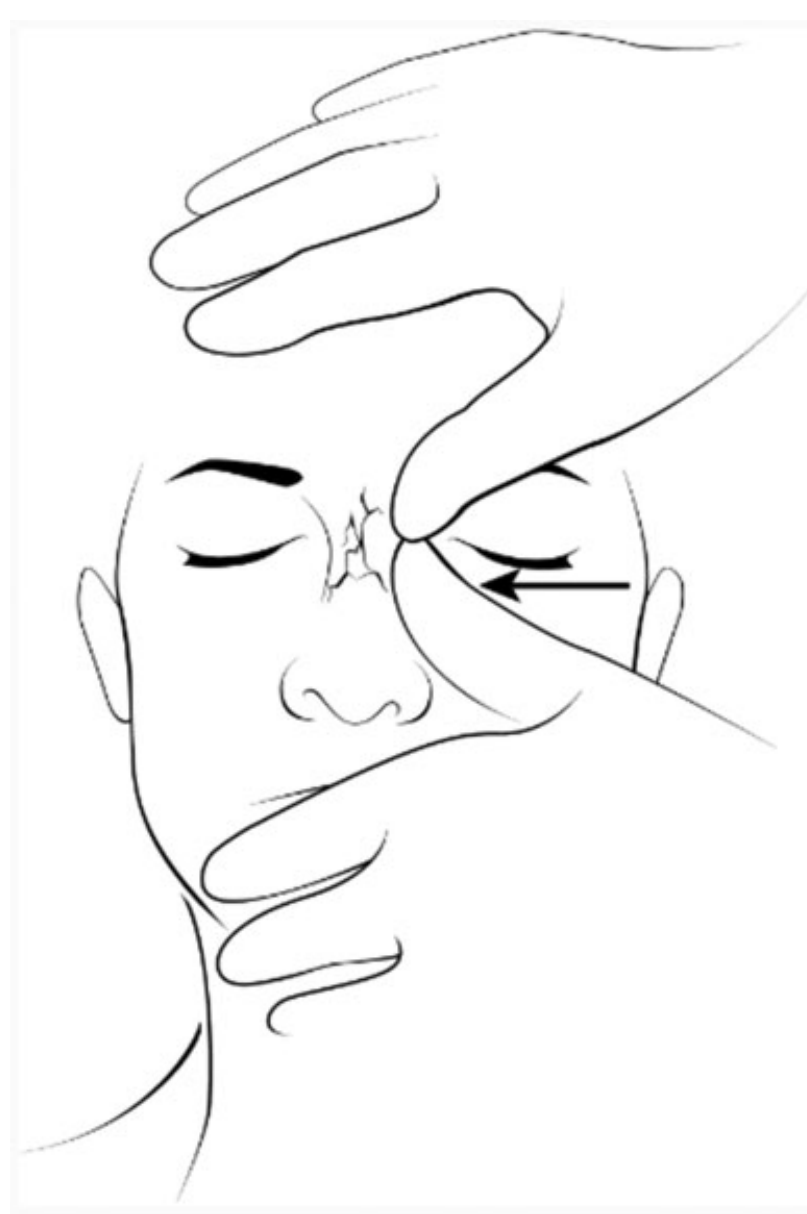

Fig. 5 Manual closed reduction can be performed with digital pressure against the nasal bones.

brief general anesthesia may be preferable in patients who are poorly cooperative or tolerant of the procedure, as some studies have shown improved functional and cosmetic out- comes, as well as patient satisfaction. ${ }^{19}$ Additionally, general anesthesia shortens the duration of the procedure considerably in most cases.

Closed nasal reduction is performed in the following fashion. Topical anesthetic and vasoconstrictor is applied through cotton pledgets in the nose. If performing under local anesthesia, cranial nerve V1 and V2 nerve blocks can be performed to improve patient tolerance of the procedure. Digital pressure along the upper third of the nose may be an effective maneuver in a mild, outfractured nasal bone deviation (-Fig. 5). However, a Boies elevator can be resourceful in addressing more significantly depressed nasal bone fracture. The length of the elevator being inserted intranasally is measured from the alar rim margin to the medial canthus to avoid overinsertion of the elevator into the skull base. The elevator is placed in the depressed nasal bone side and is placed between the nasal bones and the nasal septum intranasally (-Fig. 6A, B). The elevator is then used to lift the depressed bone out toward the surgeon and is pushed laterally to outfracture the depressed nasal bone, and at the same time, the opposite nasal bone (that is lateralized) is pushed toward the midline. It is important to mobilize the depressed nasal bone side first to create room for the contralateral, outfractured nasal bone side to medialize. Beekhuis as well as Staffel described the use of limited osteotomies if continued drift of the nose is noted after closed reduction and septoplasty. ${ }^{20,21}$ Adhesive strips and external splint are applied to the nasal skin after reduction.

After closed nasal reduction, the literature reports persistent deformity rates ranging from 14 to $50 \%$. Basheeth et al reported a persistent deformity rate of $22.5 \%$ following initial management. In their prospective series of 400 patients, only 191 patients were managed with closed nasal reduction with or without septoplasty, whereas more than $50 \%$ of patients were observed without intervention. ${ }^{14}$ In the largest prospective
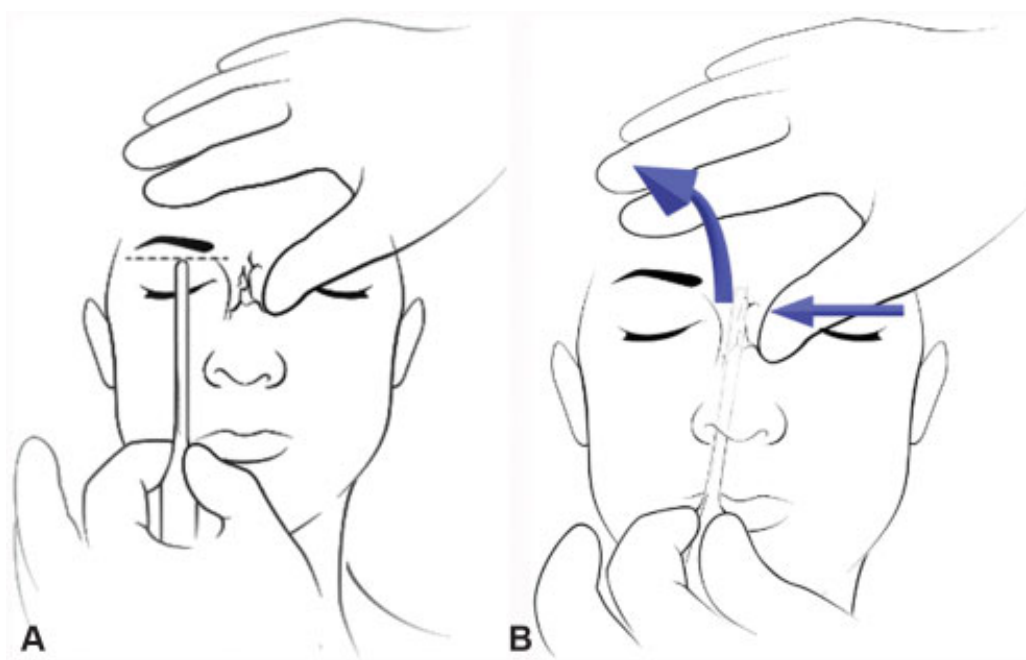

Fig. 6 (A) A Boies elevator is inserted into the naris to elevate depressed nasal bone fragments. Prior to inserting the elevator the depth of insertion should be measured along the nasal bone length from the nostril opening to avoid inserting the instrument too deeply and causing potential cerebrospinal fluid leak. (B) The elevator is placed into the concave side (depressed side) and is lifted toward the surgeon to lift the nasal bone out of the nasal cavity and also pushed laterally to outfracture the depressed segment. At the same time, once the depressed segment is lifted to create a space for the outfractured nasal bone side to move inward, a thumb placed along the convex side (outfractured side) and the outfractured nasal bone is pushed medially to bring the nasal bones into the midline. Arrows mark the direction of forces being applied. 
study of 756 patients, Murray and Maran reported a postreduction deformity rate of $41 \%{ }^{4}$

\section{Conservative Septoplasty in the Acute Setting}

To avoid persistent nasal bone deviation despite closed nasal reduction, the importance of septal correction has been widely discussed in the literature. As stated earlier, septum, nasal bones, and the upper lateral cartilages all meet together at the rhinion and form a critical structural support called the keystone area (-Fig. 2). Fry and Verwoerd's studies have demonstrated that septal fractures can cause progressive nasal deformity due to the release of locked internal stresses. ${ }^{22-24}$ Wexler showed that the nasal bones conform to the direction of the deviated septum after manipulation. ${ }^{25}$ Rohrich and Adams mention that the septum is the key structure that needs to be aligned in correcting nasal fractures to avoid secondary deformity and suggests that high reported rates of failure previously can be from unrecognized septal deformities. Ondik et al and Rohrich and Adams' postreduction deformity rate of $9 \%$ is among the lowest reported in the literature. ${ }^{10,16}$

It is important to understand that normal nasal airflow occurs primarily along the inferior nasal airway between the inferior septum and the inferior turbinates. In addition, internal and external nasal valves also have a profound impact on normal airflow, but correction of these structures are typically reserved for definitive correction surgery in a delayed fashion as extensive cartilage grafting may be required. In the acute trauma setting, both septum and the inferior turbinates can be targeted primarily as these two structures have a direct impact on normal nasal airflow. Computational fluid dynamics study has shown that anteroinferiorly located septal deviation can cause the greatest nasal obstruction, whereas medially and superiorly located septal deviation (near the skull base) has relatively limited impact on nasal airflow, highlighting the importance of correcting the caudal septal deviation and septal spurs located along the nasal floor. ${ }^{26}$ The inferior turbinates play a major role in normal flow as well. The full length of the inferior turbinates should be assessed for inferior turbinate hypertrophy, especially at the posterior end (near nasopharynx), as the posterior inferior turbinate hypertrophy can lead to nasal obstruction. ${ }^{27}$ Since unilateral nasal cavity can be considered as a cylinder that runs from the nostril opening to the nasopharynx, any obstruction that occurs due to the inferior turbinate hypertrophy, whether it occurs along the anterior, middle, or posterior segments of the inferior turbinates, may still negatively influence the nasal airflow. As such, bilateral inferior turbinates along its full length can be reduced safely at the same time in the acute trauma setting. If the septum appears tenuous in appearance with severe mucosal disruptions from trauma, it is best to minimize extensive septal mucosal dissection, as this may lead to a large septal perforation, and instead focus on removing gross septal spur that may obstruct the nasal airway and close the septal mucosal laceration, especially if there are opposing septal mucosal tears. In a situation in which the septum is severely injured with extensive septal mucosal injury, performing inferior turbinate outfracture to mobilize the lateral

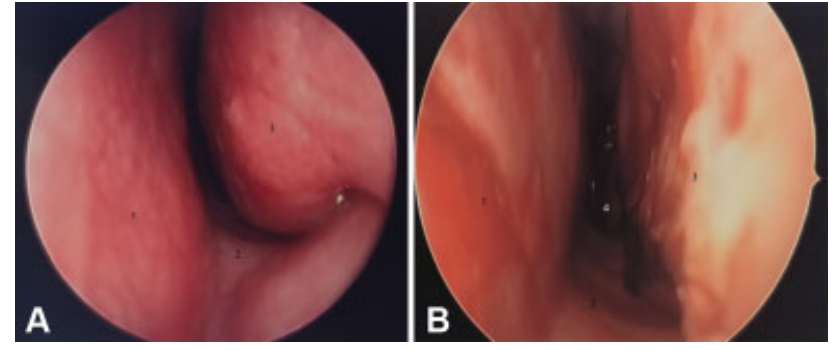

Fig. 7 (A) Endoscopic view of the left nasal airway. Before the surgery, the patient has severe left-sided inferior turbinate hypertrophy ${ }^{3}$ contacting the septum $^{1}$ and the nasal floor. ${ }^{2}$ (B) After the surgery, inferior nasal airway between the inferior septum, the nasal floor, and the inferior turbinate has been widened significantly to allow for direct visualization of the nasopharynx. ${ }^{4}$ Left inferior turbinate ${ }^{3}$ has been significantly lateralized and reduced along its full length, including the posterior head.

nasal wall away from the septum will help restore normal nasal airflow. Due to the possibility of septal perforation formation in a delayed fashion, aggressive inferior turbinate submucosal reduction is not performed as the inferior turbinates and adjacent lateral nasal wall mucosal flaps can be used as local tissue advancement flaps to repair septal perforation if it occurs in a delayed fashion. It is imperative to avoid septal perforation formation as this may also lead to nasal obstruction, and definitive corrective septoplasty with more extensive dissection may be performed in a delayed fashion once the nasal mucosa, and thus the blood supply to the septal cartilage, has fully healed. Ideally, upon completion, one should be able to easily visualize the nasopharynx in a straight line without obstruction along the nasal floor, and the maxillary crest/inferior septum should sit midline without obstructing septal spurs and the inferior turbinates should be nicely lateralized ( $\mathbf{- F i g . ~} \mathbf{7}$ ).

In the acute setting, a limited septoplasty or septal fracture reduction can be performed in the following fashion. General anesthesia is typically required. The goal in the acute setting is to minimize extensive mucoperichondrial flap dissection as this may devascularize the septal cartilage. Primary goals are to reestablish normal airflow along the inferior nasal airway and to minimize deviating force being applied to the overlying nasal bones. With that in mind, inferiorly located obstruction from septal deviation or septal spurs is identified through anterior rhinoscopy. At the same time, inferior turbinates are also assessed along its entire length to see if it will require concurrent inferior turbinate outfracture. Closed reduction of the septum may be possible using reduction forceps. If there is an isolated septal spur present, a submucoperichondrial septoplasty can be performed through Killian incision to minimize extensive mucosal dissection. Isolated septal spur along the inferior nasal airway is removed without performing the standard L-strut septoplasty to minimize mucosal dissection. If there is a severe anterior septal deviation that exists with reliable nasal mucosa present, hemitransfixion incision may be used to perform conservative septoplasty with generous preservation of L-strut (at least $1.5 \mathrm{~cm}$ in width along the dorsal and caudal septa). If there is concurrent caudal septal deviation, the senior authors (T.L. and Y.D.) use retrodissection through the hemitransfixion incision to create a soft tissue 
pocket between the medial crus, where the caudal septum can be repositioned as a tongue-in-groove fashion. This maneuver may be better reserved in a delayed corrective septoplasty as it typically requires extensive bilateral caudal mucosal flaps to be elevated and the entire caudal septum to be mobilized away from the maxillary crest attachment and repositioned into the midline position. A traction suture placed along the caudal septum and then passed through the columella skin can provide midline traction while the caudal septum is locked into the midline position with multiple transseptal sutures (-Fig. 8). ${ }^{28}$ To prevent synechiae formation from extensive mucosal swelling postoperatively and to provide additional structural support to keep the septum in midline position, Doyle or silastic splints are then placed intranasally and sutured in place for 5 to 7 days.

Recent advances in endoscopic technology have led to endoscopic instrumentation during septoplasty with good clinical results and provide improved visualization to the posterior nasal airway as well as the superior septum near the skull base if there is concurrent cerebrospinal fluid leak that requires surgical repair. Andrades et al describe a closed nasal reduction approach with endoscopic assist septoplasty in three (3.3\%) patients requiring revision septorhinoplasty. ${ }^{29}$

In summary, nasal bone fractures with concurrent severe septal deviation require conservative septoplasty or septal fracture reduction at the same time as the closed nasal reduction to minimize the risk of persistent or delayed nasal bone deviation. It is best to avoid extensive mucoperichondrium dissection during the septoplasty to preserve vascularity to the cartilage and focus on removing obstructing septal spur along the inferior septum. If there is an opposing septal mucosal laceration present, attempt should be made to reapproximate the septal mucosal linings with placement of the crushed cartilage grafting in the middle to minimize the risk of septal perforation formation. To restore normal nasal airflow, aggressive bilateral inferior turbinate outfracture can be safely performed in the acute setting, whereas conservative submucosal inferior turbinate reduction is advised in case the inferior turbinate mucosa may need to be used as a local tissue advancement flap if the patient develops a delayed septal perforation.

\section{Open Approaches in the Acute Setting}

In cases in which the nasal bone fracture cannot be properly reduced via closed methods, such as when the nasal root is involved or when in combination with LeFort, or anterior table fractures of the frontal bone, open approaches can be used. The ideal open approaches depend on the presence of laceration and the type of concurrent bony fractures present. These approaches may include the following:

- Endonasal or open rhinoplasty incision approaches if there is reliable nasal skin present without laceration (-Fig. 9).
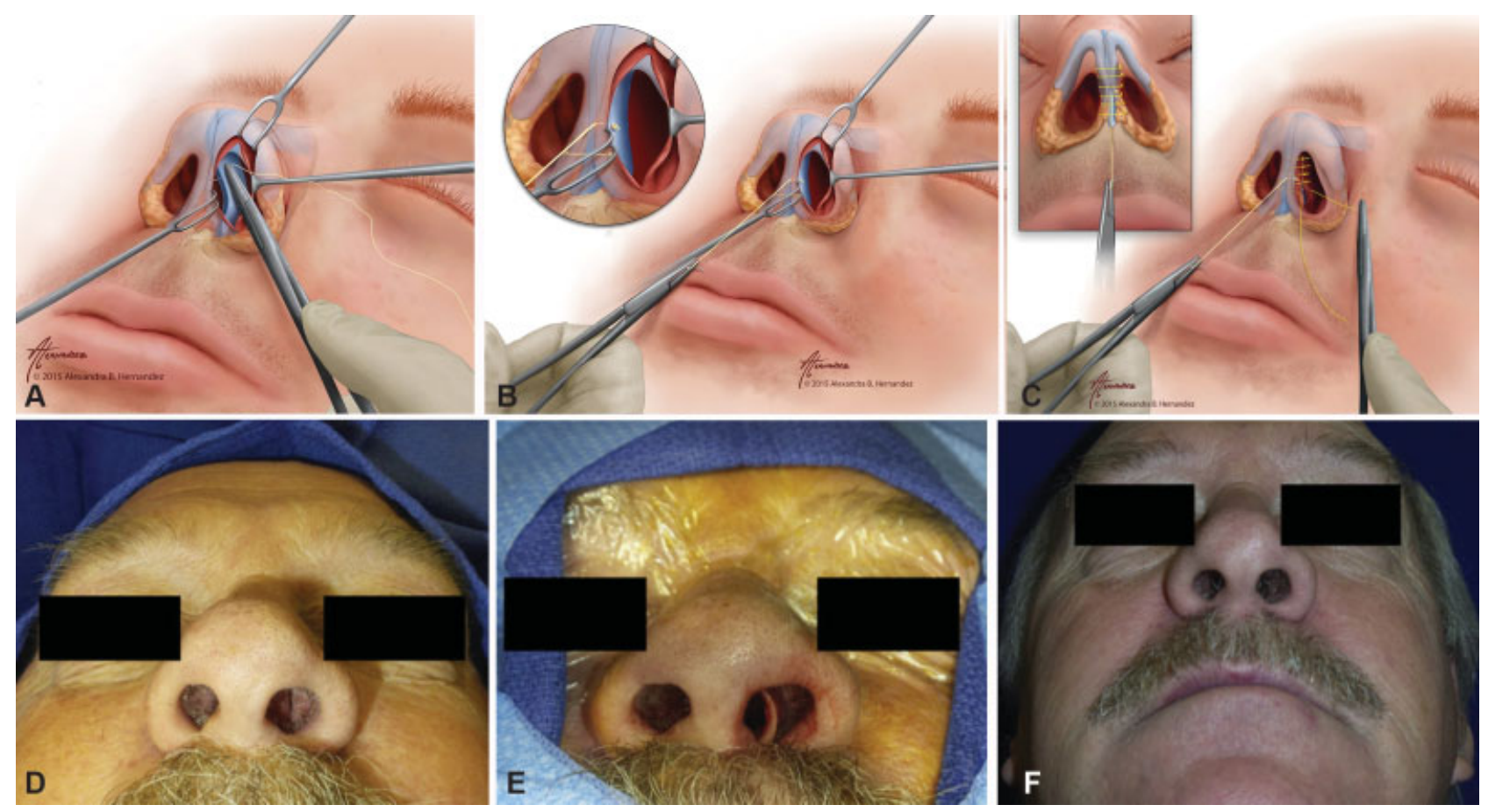

Fig. 8 (A) A soft tissue pocket is first created between the medial crus with a converse scissor through the hemitransfixion incision placed along the caudal margin of the septum. A traction suture (4-0 Vicryl) is placed at the caudal septum, then the suture is passed between the medial crura and exits through the columella. (B) Traction suture holding the septum midline by an assistant. (C) While the caudal septum is held in position at the midline, the medial crura and the surrounding mucoperichondrial flap are then quilted using multiple transseptal sutures to secure the septum into the midline position using a straight Keith needle with an absorbable suture (4-0 Chromic). Excessive medial crural foot flaring that is causing external valve narrowing can also be corrected at the same time. Transseptal sutures can also be used to close the hemitransfixion incision at the same time. (D) Left caudal septal deviation is noted preoperatively with columella asymmetry. (E) Severe caudal septal deflection is noted after bilateral mucoperichondrial dissection. (F) One-year postoperative basal view. The columella shows good symmetry and midline caudal septum 


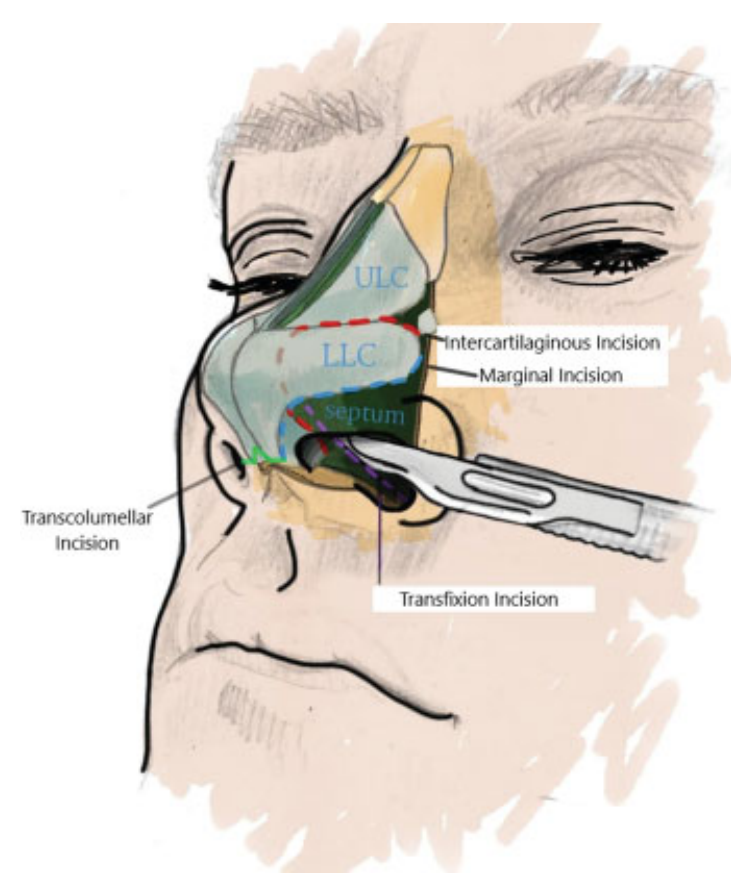

Fig. 9 Various incisions to access septum and remainder of the nose. Purple dotted line marks hemi- or full-transfixion incision. Typically, closed septoplasty is performed through hemitransfixion incision. Blue dotted line marks the marginal incision along the caudal margin of the lower lateral cartilages, which is commonly used for an open rhinoplasty approach when combined with a transcolumellar incision. Red dotted line marks the intercartilaginous incision, which can be used in endonasal rhinoplasty for access to the middle one-third for dorsal onlay as well as the upper one-third for medial osteotomy.

- Using preexisting lacerations for approach if present.

- Modified Lynch incision with a Z-plasty for isolated NOE fracture.

- Modified Lynch incision with maxillary gingival sulcus incision for concurrent LeFort II and III fractures.

- Bicoronal approach if there is a need for extensive bone grafting at the nasion that requires hardware fixation or if there is concurrent supraorbital rim or frontal sinus fractures that also require open repair

When possible, use of existing lacerations limits additional incisions and scars in the patient to optimize cosmesis. If a laceration is present, an open rhinoplasty incision should not be used as this may fully devascularize the tenuous nasal skin. Endonasal rhinoplasty incision, such as intercartilaginous incision, can be used for dorsal onlay graft placement along the upper two-thirds to further augment the nasion or to correct mild saddle-nose deformity, and it can also be used to perform medial osteotomy. The dorsal onlay graft derived from either an iliac/calvarial bone graft or ear/rib cartilage graft can be inserted in a midline subperichondrial/subperiosteal dissection pocket without the need for hardware plating. If there is a concern for infection, it is again best to avoid extensive cartilage grafting.

For repair of an NOE fracture without a laceration, the traditional gull-wing incision should be avoided as it tends to leave an unacceptable appearing scar. The modified Lynch incision provides a direct open access to the NOE region. The risk of developing a webbed scar along the medial canthus region can be minimized by using a z-plasty modification described by Esclamado and Cummings. ${ }^{30}$ When there is concurrent LeFort II or III fractures, a modified Lynch incision can be combined with a maxillary gingival sulcus incision.

A bicoronal incision may be useful in cases of severe comminution when a large bone graft must be secured with hardware to restore the nasal contour along the upper two-thirds. However, a smaller bone graft or a dorsal onlay cartilage graft can be instead placed using an endonasal, intercartilaginous incision that is more cosmetically favorable. Furthermore, bicoronal incision is typically reserved for situations in which supraorbital rim or frontal sinus fractures must also be repaired. A major limitation of the bicoronal incision is that the inferior extent of the approach is at the nasion, limiting access to the middle third of the nose. If needed, the bicoronal approach may need to be combined with an open rhinoplasty approach if the entire length of the nose needs to be accessed. Incision alopecia and temporal hollowing (if dissected through the temporal fat pad) are potential complications of the bicoronal approach. Temporal hollowing can be minimized if the deep temporalis fascia (fascia on top of the temporalis muscle) is not violated and instead the dissection is performed immediately on top of the deep temporalis fascia while remaining deep to the loose areolar tissue and the temporoparietal fascia.

\section{Open Septorhinoplasty in Acute Trauma}

Severe septal fractures, comminuted nasal bone fractures, resulting in significant destabilization of the nasal framework are indications for open surgical approaches. Mondin et al's indications for open surgical reduction include extensive fracture or dislocation of nasal bones and septum, nasal pyramid deviation greater than half the width of the nasal bridge, open septal fractures, and caudal septal fractures. ${ }^{31}$ McCullough described dorsal hump reductions being amenable to treatment with bilateral osteotomies in the setting of primary nasal fracture repair. ${ }^{32}$ However, if there is an extensive nasal skin laceration present, it is best to avoid using a traditional open rhinoplasty approach (marginal + transcolumellar incision) and let the skin envelope heal first before considering an open approach. Instead, an existing laceration should be used for approach with limited skin flap elevation, just enough to see the fracture and to place the hardware. Additional skin incisions that can deglove the nasal skin should be avoided. As such, senior authors (T.L and Y.D.) typically defer open septorhinoplasty for a delayed definitive correction surgery at 1 to 6 months post trauma depending on the severity of cosmetic and functional problems and the potential for infection.

Due to the significant skin and soft tissue swelling that can interfere with assessing outcome during the surgery, the use of primary open septorhinoplasty for nasal bone fractures is uncommon but reported. With careful patient selection, some authors actually advocate early open procedures as patient outcomes are better than closed reduction results. ${ }^{33}$ Staffel concluded that certain rhinoplasty procedures can be safely employed in the setting of acute nasal bone fracture. ${ }^{21}$ $\mathrm{Kim}$ et al demonstrated in a retrospective series of 56 
patients that simultaneous cosmetic rhinoplasty was relatively safe in open reduction of nasal bone fractures, with one revision case of saddle nose with significant septal fracture. ${ }^{34}$ Another group focused on a retrospective analysis of patients with existing nasal deformity prior to nasal bone fracture. Of 45 patients who underwent open rhinoplasty within 4 weeks of fracture, spreader grafts and extracorporeal septoplasty were used predominantly in different fracture patterns, with a revision rate of $4.4 \%{ }^{7}$

Proponents of acute septorhinoplasty approach stress that direct visualization of the nasal bones allows for more accurate reduction. In the Asian population, patients may request dorsal augmentation concurrently, and this can be addressed safely at the time of nasal fracture reduction per Kim et al. ${ }^{34}$ Challenges include fibrosis and difficult dissection of fractured nasal bone segments.

Ondik et al showed revision rates, patient satisfaction, and outcomes to be similar in closed and open reduction groups. However, this retrospective study focused on various fracture patterns, and the majority of the open approach group were associated with higher level fracture patterns. ${ }^{16}$ No studies to date have compared closed reduction with open repair for similar fracture patterns. Overall, there is likely a subset of severe nasal fracture patients who are candidates for primary open repair, but the majority of them appear to be manageable with closed reduction. Most can agree that if concurrent extensive nasal laceration or infection is present, the laceration should be used for open approach, and the corrective open septorhinoplasty should be performed in a delayed fashion once the skin envelope or residual infection has fully resolved.

In cases of concurrent NOE, LeFort, and frontal sinus fractures, open approaches to address reduction and fixation are well established. The details of these fracture repairs are beyond the scope of this paper, but, nonetheless, this subset of nasal trauma patients may also require revision septorhinoplasty following initial repair.

\section{Patient Considerations}

Studies have found that those who undergo nasal fracture repair have lower postoperative expectations if they do not have a history of previous cosmetic rhinoplasty. Reilly et al showed that five of six patients requiring revision following open reduction had previous cosmetic rhinoplasty. ${ }^{35}$ There may be some intrinsic expectations that lead this subgroup of patients to require revision procedures. Questionnaire data are often confounded by patients' lack of desire for undergoing secondary revision surgery and may partially explain the disparity in patient versus surgeon satisfaction following closed reduction (62-91\% vs. 21-65\%). ${ }^{21}$ Hung et al reported a retrospective study of 62 patients who underwent closed nasal reduction. Eighteen patients were unsatisfied with their result, but only 11 of these requested revision surgery. Of those who were satisfied, seven requested further surgery. This study had an overall secondary surgery rate of $29 \% .^{1}$ These findings are in line with Li et al's population data reporting that patients with previous nasal deformity or obstruction are at an increased risk for revision septorhinoplasty. ${ }^{5}$ These patients may inherently be more aware and fixate on the subtler details of their nose compared with the general population and may be prone to a higher rate of dissatisfaction. Preoperative deformities are thus important to assess, when possible, and can be assessed with preinjury photos.

\section{Revisions}

Following primary repair of the nasal fracture through whichever approach, patients require follow-ups at least 1 to 3 months postoperatively for any residual cosmetic or functional deficits. The secondary treatment that can be offered can be broken down based on functional or cosmetic complaints. In most cases, functional concerns should be prioritized before cosmetic concerns, but in many instances, they can be addressed concurrently. For primarily functional concerns, medical management includes optimization of nasal mucosa and inferior turbinate hypertrophy with nasal steroid sprays and routine nasal irrigation rinses.

If the nasal obstruction persists despite 1 to 3 months of conservative medical therapy, the patient may benefit from corrective nasal airway surgery using either endonasal or open septorhinoplasty with inferior turbinate reduction as is routinely performed in nontraumatic nasal airway surgery patients. Once a nasal skin laceration or infection has fully resolved with time, there is no serious risk of nasal cartilage being devascularized with extensive skin and mucosal dissection that is commonly seen in definitive septorhinoplasty. Cartilage grafting can be performed reliably in a delayed corrective surgery.

The components affecting the nasal obstruction must be properly assessed preoperatively, as is performed for any nontraumatic nasal airway surgery. In particular, septal deviation along the caudal aspect and along the maxillary crest near the nasal floor should be corrected to optimize normal airflow that occurs along the inferior airway. Any deviation along the maxillary crest is removed using hemitransfixion incision. Caudal septal deviation can also be corrected using the traction suture technique in a tonguein-groove fashion. ${ }^{28}$ Similarly, inferior turbinate hypertrophy along its full length should be reduced using submucosal reduction techniques and outfracture to lateralize the inferior turbinates to widen the inferior nasal airflow. If there is a septal perforation present, closure of the septal perforation should be of top priority, as it will lead to nasal obstruction from recirculation. In addition, dynamic internal and external nasal valve collapse should be evaluated and addressed using various grafting options to strength the valve region. Asymmetric middle one-third collapse with or without concurrent internal nasal valve collapse can be addressed using a wide range of techniques including asymmetrically placed spreader grafts, butterfly suture technique, and a dorsal onlay augmentation. If there is a severe fracture along the dorsal L-strut, it may require extended spreader grafts to rebuild the L-strut to avoid a saddle-nose deformity (-Fig. 10). Lastly, nasal tip deviation with undesirable rotation or projection can be corrected during the revision surgery using a multitude of rhinoplasty techniques (caudal septal extension graft, columellar strut graft, caudal septum repositioning [ - Fig. 8], lateral strut grafts, rim grafts, lateral 


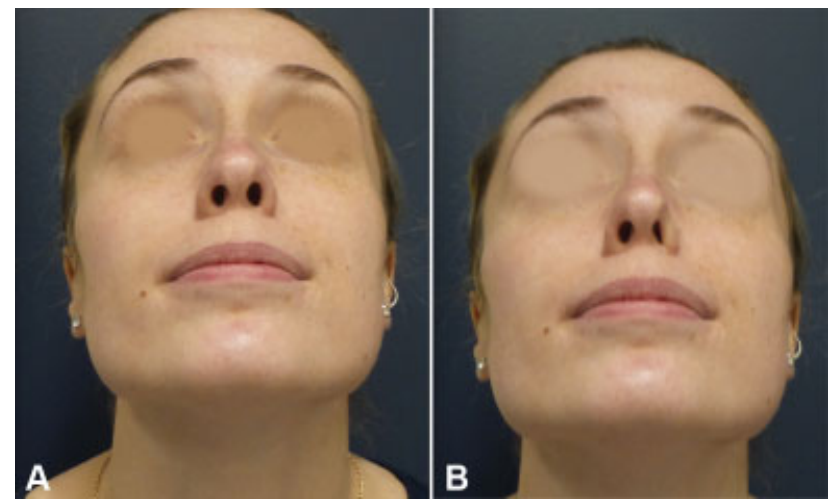

Fig. 10 (A) Patient at rest without inspiration, basal view demonstrating symmetric nostrils without any obvious valve collapse. (B) The basal view on inspiration demonstrating collapse of the left external nasal valve.

crural steal, medial crural steal, intra- or interdomal sutures, shield graft, etc.). For upper one-third nasal bone deviation, medial and lateral osteotomies can be performed, and if additional volume is desired to optimize contour, bone graft or cartilage graft can be placed. In individuals with thick skin, it is common for a laceration to cause extreme thinning of the skin, giving the illusion of saddle-nose deformity even when the L-strut is well maintained. In such a situation of undesirable thin scar affecting the profile contour, placement of dorsal onlay grafts at the laceration site, such as finely crushed cartilage, fascia graft, or acellular dermal matrix (such as Alloderm; Allergan), may be an option to provide loss of volume in the subcutaneous tissue plane. The multitude of corrective maneuvers that exists with corrective open septorhinoplasty is outside the scope of this paper. This corrective procedure is typically delayed at least 3 to 6 months after the initial injury and repair to allow bone and cartilaginous healing, as well as minimization of facial edema to allow a more precise surgical result.

Following the management strategy and resolution of soft tissue edema, as well as discussion of patient expectations, minor cosmetic deformities without functional airway obstruction may be corrected with injectable fillers. This may be a suitable management strategy in patients who are not fully satisfied with their results but do not want to pursue further surgical treatment.

For demonstration, we present a case of untreated septal hematoma developing a delayed septal abscess after a motor
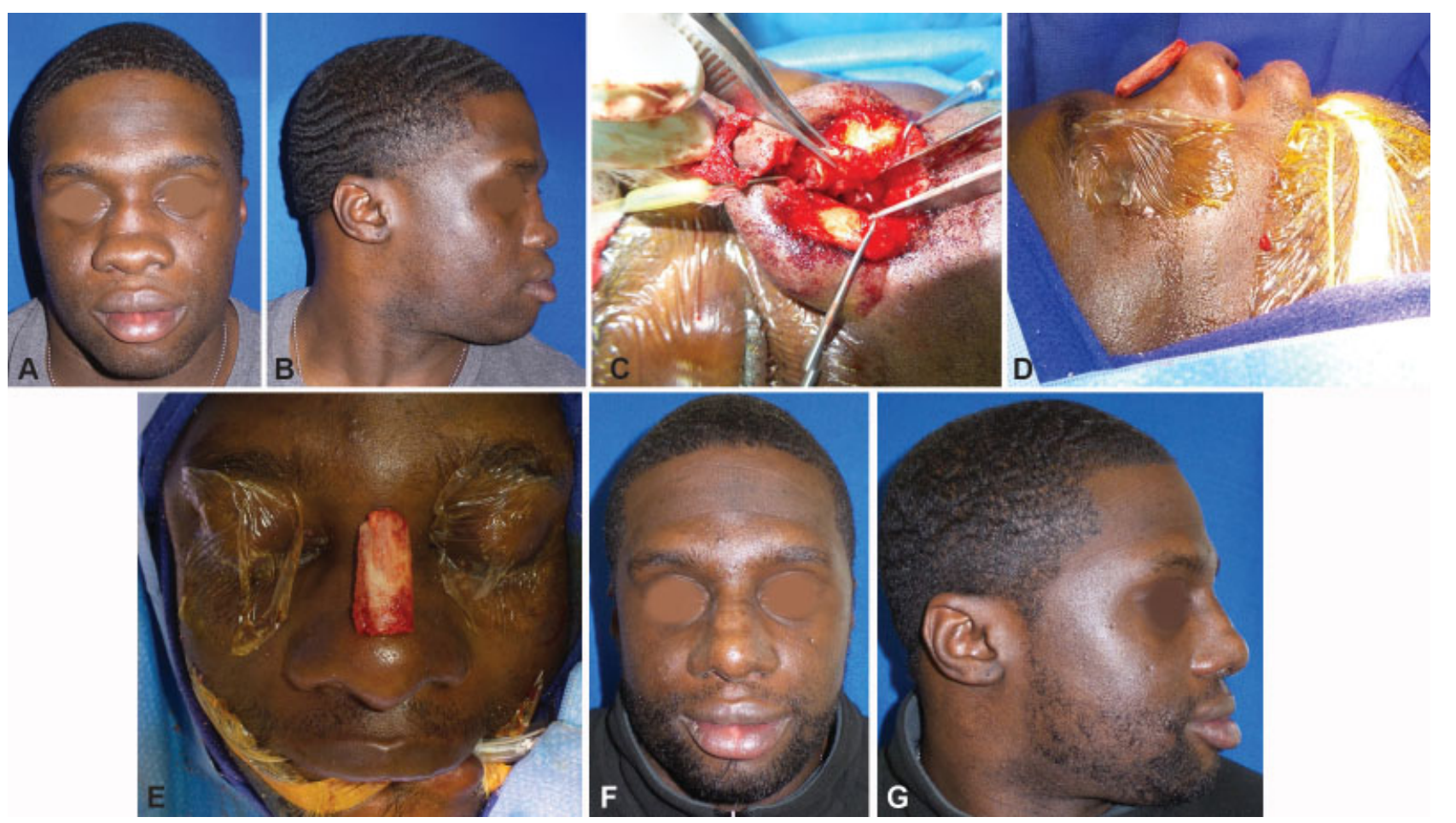

Fig. 11 (A) The patient presented with infected septal hematoma at 1 month after trauma. He underwent immediate septal hematoma drainage with a drain placement and oral antibiotics treatment. The drain was removed after 1 week and was treated with antibiotics for a total of 3 weeks. Due to the severe infection, final reconstruction was delayed for 3 months to allow for complete resolution of septal cartilage infection. The patient complained of severe bilateral nasal obstruction, and the preoperative frontal view is shown. (B) The sagittal view shows severe saddle-nose deformity along the middle one-third due to total septal collapse. (C) An open septorhinoplasty approach was used. The septum showed severe scarring with total destruction of L-strut with entirely missing caudal septum. (D) For nasal obstruction correction, bilateral inferior turbinate reduction and septoplasty were performed. The remaining septum at the keystone area was preserved. The entire septal L-strut was reconstructed using rib cartilage to create bilateral extended spreader grafts that were secured to the remaining septum at the keystone area and placed between the bilateral upper lateral cartilages. The extended spreader grafts were then secured to a large columellar strut graft located between the medial crura. The nasal tip shows improved projection and rotation along the lower one-third. (E) To improve contour along the upper two-thirds, an iliac crest bone graft was shaped as a dorsal onlay graft and placed on top of the extended spreader grafts and the nasal bones to further correct the saddle-nose deformity. (F) At 1 year postoperative, the patient reported good bilateral nasal breathing. The frontal view is shown. (G) The sagittal view shows an excellent profile with correction of the saddle-nose deformity. 
vehicle accident. The patient went on to develop saddle-nose deformity and bilateral nasal obstruction. Initially, the septal abscess was drained and treated with antibiotics. A corrective delayed open septorhinoplasty approach was performed 3 months after the infection had completely cleared. The patient required total septal L-strut reconstruction with bilateral extended spreader grafts and large columellar strut graft derived from a rib cartilage graft. In addition, iliac bone graft was used to augment the upper two-thirds to provide an optimal nasal profile (-Fig. 11). A large rib cartilage graft can also be used as a large dorsal onlay as an alternative option.

\section{Conclusion}

Proper management of acute nasal trauma is important in minimizing secondary deformities and impaired function. Closed nasal reduction is typically used in the acute setting for deviated nasal bone fracture. An ideal algorithm with routine assessment of septal involvement may improve failure rates of closed nasal reduction. For severe septal fractures, conservative and limited septoplasty should be performed with minimal mucoperichondrial flap dissection while avoiding septal perforation formation. Careful patient selection is advised if a traditional open rhinoplasty approach is used in the acute setting. Primary use of open septorhinoplasty of isolated nasal fractures in the acute setting is debated. Generally, a definitive open septorhinoplasty with cartilage grafting can be performed more reliably in a delayed fashion once the skin envelope, swelling, cartilage injury, and infection issues have fully resolved with time. More extensive upper and midfacial trauma with concurrent nasal fractures may require transcutaneous open approaches with rigid fixation in the acute setting.

Conflicts of Interest

None declared.

\section{References}

1 Hung T, Chang W, Vlantis AC, Tong MC, van Hasselt CA. Patient satisfaction after closed reduction of nasal fractures. Arch Facial Plast Surg 2007;9(01):40-43

2 Watson DJ, Parker AJ, Slack RW, Griffiths MV. Local versus general anaesthetic in the management of the fractured nose. Clin Otolaryngol Allied Sci 1988;13(06):491-494

3 Waldron J, Mitchell DB, Ford G. Reduction of fractured nasal bones; local versus general anaesthesia. Clin Otolaryngol Allied Sci 1989;14(04):357-359

4 Murray JA, Maran AG. The treatment of nasal injuries by manipulation. J Laryngol Otol 1980;94(12):1405-1410

5 Li K, Moubayed SP, Spataro E, Most SP. Risk factors for corrective septorhinoplasty associated with initial treatment of isolated nasal fracture. JAMA Facial Plast Surg 2018;20(06):460-467

6 Stevens MR, Emam HA. Applied surgical anatomy of the nose. Oral Maxillofac Surg Clin North Am 2012;24(01):25-38

$7 \mathrm{Kim}$ KS, Lee HG, Shin JH, Hwang JH, Lee SY. Trend analysis of nasal bone fracture. Arch Craniofac Surg 2018;19(04):270-274

$8 \mathrm{Kim}$ JE, Park HS, Yoon CH, Kim HJ. Analysis of nasal septal fracture combined in nasal bone fracture using CT. JKorean Soc Plast Reconstr Surg. 1998;25:852
9 Lee M, Inman J, Callahan S, Ducic Y. Fracture patterns of the nasal septum. Otolaryngol Head Neck Surg 2010;143(06):784-788

10 Rohrich RJ, Adams WP Jr. Nasal fracture management: minimizing secondary nasal deformities. Plast Reconstr Surg 2000;106(02): 266-273

11 Gilles H, Kilner T. Modern technique in treatment. Lancet 1929; 1:147-149

12 Stranc MF, Robertson GA. A classification of injuries of the nasal skeleton. Ann Plast Surg 1979;2(06):468-474

13 Murray JA. Management of septal deviation with nasal fractures. Facial Plast Surg 1989;6(02):88-94

14 Basheeth N, Donnelly M, David S, Munish S. Acute nasal fracture management: a prospective study and literature review. Laryngoscope 2015;125(12):2677-2684

15 Hoffmann JF. An algorithm for the initial management of nasal trauma. Facial Plast Surg 2015;31(03):183-193

16 Ondik MP, Lipinski L, Dezfoli S, Fedok FG. The treatment of nasal fractures: a changing paradigm. Arch Facial Plast Surg 2009;11 (05):296-302

17 Lee DH, Jang YJ. Pediatric nasal bone fractures: does delayed treatment really lead to adverse outcomes? Int J Pediatr Otorhinolaryngol 2013;77(05):726-731

18 Yoon HY, Han DG. Delayed reduction of nasal bone fractures. Arch Craniofac Surg 2016;17(02):51-55

19 Al-Moraissi EA, Ellis E III. Local versus general anesthesia for the management of nasal bone fractures: a systematic review and meta-analysis. JOral Maxillofac Surg 2015;73(04):606-615

20 Beekhuis GJ. Nasal fractures. Trans Am Acad Ophthalmol Otol 1970;74:1058-1059

21 Staffel JG. Optimizing treatment of nasal fractures. Laryngoscope 2002;112(10):1709-1719

22 Verwoerd CD. Present day treatment of nasal fractures: closed versus open reduction. Facial Plast Surg 1992;8(04):220-223

23 Fry HJ. Interlocked stresses in human nasal septal cartilage. $\mathrm{Br} \mathrm{J}$ Plast Surg 1966;19(03):276-278

24 Fry H. Nasal skeletal trauma and the interlocked stresses of the nasal septal cartilage. Br J Plast Surg 1967;20(02):146-158

25 Wexler MR. Reconstructive surgery of the injured nose. Otolaryngol Clin North Am 1975;8(03):663-677

26 Garcia GJ, Rhee JS, Senior BA, Kimbell JS. Septal deviation and nasal resistance: an investigation using virtual surgery and computational fluid dynamics. Am J Rhinol Allergy 2010;24(01):e46-e53

27 Lee TS, Goyal P, Li C, Zhao K. Computational fluid dynamics to evaluate the effectiveness of inferior turbinate reduction techniques to improve nasal airflow. JAMA Facial Plast Surg 2018;20 (04):263-270

28 Indeyeva YA, Lee TS, Gordin E, Chan D, Ducic Y. Traction suture modification to tongue-in-groove caudal septoplasty. Int J Oral Maxillofac Surg 2018;47(02):180-183

29 Andrades P, Pereira N, Borel C, Rocha L, Hernández R, Villalobos R. A new approach to nasoseptal fractures: submucosal endoscopically assisted septoplasty and closed nasal reduction. JCraniomaxillofac Surg 2016;44(10):1635-1640

30 Esclamado RM, Cummings CW. Z-plasty modification of the Lynch incision. Laryngoscope 1989;99(09):986-987

31 Mondin V, Rinaldo A, Ferlito A. Management of nasal bone fractures. Am J Otolaryngol 2005;26(03):181-185

32 McCullough EG. Nasal Plastic Surgery. Philadelphia, PA: WB Saunders Co.; 1994:230

33 Clark WD, Stiernberg CM. Early aggressive treatment of nasal fractures. Ear Nose Throat J 1986;65(10):481-483

$34 \mathrm{Kim} \mathrm{JH}$, Lee JW, Park CH. Cosmetic rhinoseptoplasty in acute nasal bone fracture. Otolaryngol Head Neck Surg 2013;149(02):212-218

35 Reilly MJ, Davison SP. Open vs closed approach to the nasal pyramid for fracture reduction. Arch Facial Plast Surg 2007;9(02):82-86 